\title{
Contralateral acupuncture versus ipsilateral acupuncture in the rehabilitation of post-stroke hemiplegic patients: a systematic review
}

\author{
Mi-kyung Kim², ${ }^{1,2}$ Tae-Young Choi ${ }^{3}$, Myeong Soo Lee ${ }^{3}$, Hyangsook Lee ${ }^{4}$, Chang-ho Han ${ }^{1,2^{*}}$
}

\begin{abstract}
Background: Contralateral acupuncture (CAT) involves inserting needles in the meridian on the side opposite the disease location and is often used in post-stroke rehabilitation. The aim of this systematic review is to summarize and critically evaluate the evidence for and against the effectiveness of CAT for post-stroke rehabilitation as compared to ipsilateral acupuncture (IAT).

Methods: Seventeen databases were searched from their inceptions through June 2010. Prospective clinical trials were included if CAT was tested as the sole treatment or as an adjunct to other treatments for post-stroke rehabilitation and compared to IAT.

Results: Eight randomized clinical trials (RCTs) met our inclusion criteria. Four of them reported favorable effects of CAT compared to IAT for at least one outcome. A meta-analysis showed superior effects of CAT compared to IAT on recovery rate $(n=361$; risk ratio $(R R), 1.12 ; 95 \%$ confidence intervals $(C l s), 1.04$ to $1.22, P=0.005)$. Subgroup analysis also showed favorable effects of using CAT on patients with cerebral infarction ( $n=261 ; R R, 1.15 ; 95 \%$ Cls, 1.04 to $1.27, P=0.006$ ). Further analysis including patients with cerebral infarction and intracranial hemorrhage, however, failed to show these advantages ( $n=100 ; R R, 1.11 ; 95 \% \mathrm{Cls}, 0.85$ to $1.46, \mathrm{P}=0.43$ ).

Conclusion: The results of our systematic review and meta-analysis suggest that there is limited evidence for CAT being superior to IAT in the treatment of cerebral infarction. The total number of RCTs included in our analysis was low, however, and the RCTs included had a high risk of bias. Future RCTs appear to be warranted.
\end{abstract}

\section{Background}

Stroke is one of the most common causes of death in the world. Despite the considerable benefits of organized stroke care, many stroke survivors remain moderately or severely disabled $[1,2]$. In Western medicine, no single form of complementary or alternative medicine (CAM) is commonly used to manage post-stroke rehabilitation or recovery [3]. In East-Asian countries, however, acupuncture and herbal medicine are widely used [3]. A recent survey reported that $46 \%$ of stroke patients use some form of CAM such as herbal medicine, acupuncture-type treatments or chiropractic treatments [4]. Contralateral acupuncture (CAT), also called opposite needling or crossing needling, is a needling technique where

\footnotetext{
*Correspondence: hani@dgu.ac.kr

'Department of Korean Internal Medicine, Dongguk University IIsan Hospital, Goyang, South Korea

Full list of author information is available at the end of the article
}

acupuncture points on the right side are selected for diseases on the left and vice versa. The technique is commonly used and usually preferred to ipsilateral acupuncture (IAT) for treating post-stroke hemiplegia or sciatica [5]. Several studies have shown effects of CAT on pain [6,7], dizziness [8], herpes zoster [9], and stroke [10].

Although poor methodological quality and possibility of publication bias limits the strength of recommendation, the most recently published meta-analysis suggests that acupuncture may be an effective treatment for post-stroke rehabilitation [11]. In practice, CAT is often used as a treatment in post-stroke rehabilitation for hemiplegic patients in East-Asian Medicine hospitals and clinics. Thus, it seems pertinent to evaluate the effectiveness of CAT in post-stroke rehabilitation. To date, no systematic review has been performed on this topic. The aim of this systematic review, therefore, was to summarize and critically evaluate the evidence for or 
against the effectiveness of CAT for post-stroke rehabilitation of hemiplegic patients as compared to IAT.

\section{Methods \\ Databases}

The following databases were searched from their inceptions through June 2010: MEDLINE, EMBASE, CINAHL, AMED, Cochrane Central Register of Controlled Trials, Cochrane Database of Systematic Reviews, Database of Abstracts of Reviews of Effects, Cochrane Methodology Register, a Chinese medical database (CNKI), three Japanese medical databases (Journal Archive, Science Link Japan, and Japan Science \& Technology link), 6 Korean medical databases (The Research Information Service System, Korean Studies Information, DBPIA, Korea Institute of Science and Technology Information, KoreaMed, and Korean National Assembly Library), and 4 major Korean traditional medical journals (Journal of Korean Oriental Internal Medicine, Journal of Oriental Rehabilitation Medicine, Journal of Sasang Constitutional Medicine and Journal of Korean Oriental Medicine). The following search terms were used: [(“Contralateral acupuncture" OR "Contralateral needling" OR "Healthy side acupuncture" OR "Healthy side needling" OR "Opposite side acupuncture" OR "Opposite side needling") AND (stroke OR apoplexy OR cva OR cerebrovascular attack OR cerebrovascular accident OR cerebrovascular* OR cerebral infarction OR cerebral hemorrhage OR cere$\left.\mathrm{bral}^{*}\right)$. Furthermore, the references from all located articles were manually searched for additional relevant articles.

\section{Study selection}

Prospective randomized clinical trials (RCTs) comparing the clinical effects of CAT to those of IAT were included. The studies involving post-stroke hemiplegic patients who were diagnosed clinically and/or by brain computed tomography (CT) scan or brain magnetic resonance imaging (MRI), including patients with cerebral infarction, intracerebral hemorrhage, cerebral embolism, or unclassified stroke, were included. To be included, acupuncture treatment on the affected side should be compared with that on the unaffected side. Trials where concomitant treatments were given were also included if they were given to both acupuncture and control groups. No language restrictions were imposed on study selection, and dissertations and abstracts were included provided they contained sufficient detail.

\section{Data extraction, quality control, and assessment of the risk of bias}

All articles were read by two independent reviewers (MKK, TYC) who extracted data from each article according to predefined criteria. The risk of bias was assessed using the assessment tool for 'risk of bias' from the Cochrane Handbook for Systematic Reviews of Interventions [12]. The following characteristics were assessed: (1) Was the allocation sequence adequately generated? (2) Was allocation adequately concealed? (3) Was knowledge of the allocated interventions adequately prevented during the study? (4) Were incomplete outcome data adequately addressed? (5) Are reports of the study free of suggestion of selective outcome reporting? (6) Was the study free of other problems that could put it at a risk of bias?. Our review used 'Y, U, N' as keys of the judgments; the answer 'Yes' indicated a low risk of bias (Y), 'Unclear' indicated that a risk of bias is uncertain $(\mathrm{U})$, and the answer 'No' indicated a high risk of bias $(\mathrm{N})$.

Patient blinding was assumed when the control intervention was indistinguishable from acupuncture, even if the word "blinding" was not used in the report. Given that it is virtually impossible to blind therapists to the types of acupuncture, we assessed patient and assessor blinding separately. Disagreements were resolved by discussion between the two reviewers (MSL, HL). There were no disagreements between the two reviewers about the results.

\section{Data synthesis}

To summarize the effects of acupuncture on patient outcomes as compared to baseline, we estimated weighted mean differences (WMD) or standardized mean differences (SMD) and 95\% confidence intervals (CIs) for each study using the Cochrane Collaboration's software (Review Manager [RevMan] Version 5.0 for Windows. Copenhagen: The Nordic Cochrane Centre). Relative risk (RR) and 95\% CIs were also calculated. The variance of change was determined using a correlation factor of 0.5. When appropriate (i.e., when excessive statistical heterogeneity did not exist), we pooled the data across studies using a random effects model. The chisquare, $\operatorname{tau}^{2}$ and Higgins $\mathrm{I}^{2}$ tests were used to assess heterogeneity.

\section{Results}

\section{Study description}

The literature searches identified 119 potentially relevant articles, 8 of which were included in this review (Figure 1). Key data from the 8 included RCTs are summarized in Table 1[10,13-19]. Seven of the included trials were from China [13-19], and one was from Korea [10]. In addition, six of the included trials [10,13-17] followed a two-arm parallel group design, while two employed four-arm parallel group design $[18,19]$. The devices used for treatment were either electroacupuncture (EA) $[14,15]$ or manual acupuncture [10,13,16-19]. 


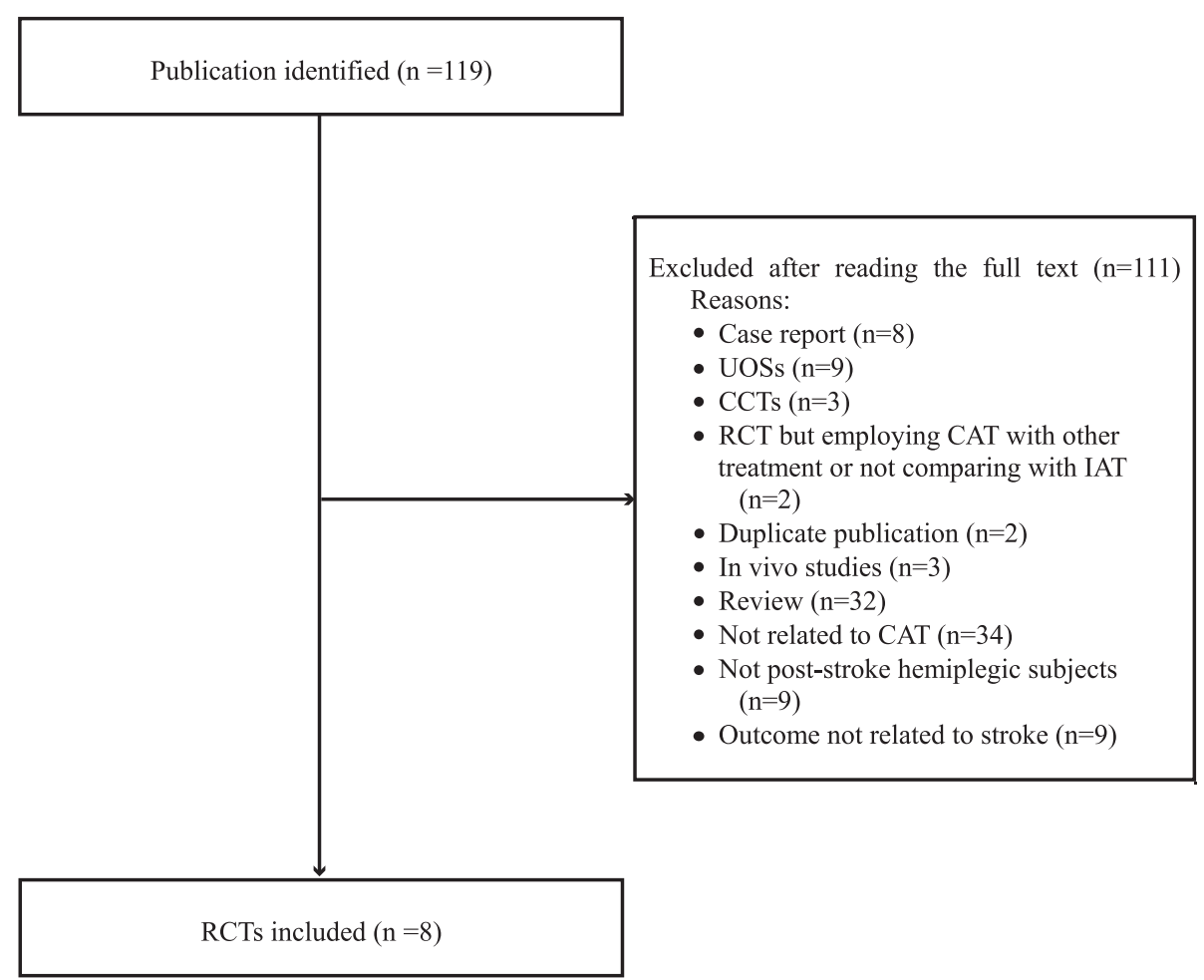

Figure 1 Flow chart of the study selection process. UOS: uncontrolled observational study; CCT: controlled clinical trial; RCT: randomized clinical trial; CAT: contralateral acupuncture; IAT: ipsilateral acupuncture.

In one trial, a big size needle, i.e. 0.4-1.6 $\mathrm{mm} \times 150-500$ $\mathrm{mm}$, penetrating from one acupuncture point through another in CAT group was compared with IAT using filiform needles [15]. All of the included trials used CT scans and MRI to diagnose stroke. Four of the RCTs [13-16] included subjects with cerebral infarction, while the other four [10,17-19] included subjects with either cerebral infarction or intracranial hemorrhage.

\section{Risk of Bias}

Risk of bias assessment is shown in Table 1. Three of the included RCTs described the sequence generation [17-19] and they all used proper methods [10,17-19]. It was not clear whether group assignment was adequately concealed in any of the included trials. All of the included trials were rated as ' $U$ ' or ' $N$ ' for patient blinding. and two studies were rated as ' $\mathrm{Y}$ ' for outcome assessor blinding $[10,18]$. For incomplete outcome data reporting, one study did not clearly reported how many patients were analyzed [14].

\section{Outcome Measures}

\section{Response rate}

Six of the included trials examined the effects of CAT as compared to IAT on response rate [13-18]. One trial showed a favorable effect of CAT $[16,18]$, while the others failed to do so. A meta-analysis, however, showed that CAT had superior effects compared to IAT on response rate $(\mathrm{n}=361$; $\mathrm{RR}, 1.12 ; 95 \%$ CIs, 1.04 to 1.22 , $\mathrm{P}=0.005$; heterogeneity: $\chi^{2}=2.71, \mathrm{P}=0.75, \mathrm{I}^{2}=0 \%$ Figure 2A). Subgroup analysis also showed favorable effects of CAT for patients with cerebral infarction ( $\mathrm{n}=261$; RR, 1.15 ; 95\% CIs, 1.04 to $1.27, \mathrm{P}=0.006$; heterogeneity: $\chi^{2}=0.65, \mathrm{P}=0.88, \mathrm{I}^{2}=0 \%$ ) [13-16]. Further analysis including patients with cerebral infarction and intracranial hemorrhage, however, failed to find these favorable effects $(\mathrm{n}=100 ; \mathrm{RR}, 1.11 ; 95 \%$ CIs 0.85 to $1.46, \mathrm{P}=0.43$; heterogeneity: $\chi^{2}=1.78, \mathrm{P}=0.18$, $\left.\mathrm{I}^{2}=44 \%\right)[17,18]$.

\section{Activities of Daily Living (ADL)}

Three of the RCTs assessed the effects of CAT on ADL compared to IAT $[10,17,19]$. One trial showed favorable effects of CAT [17], while the others did not [10,19]. A meta-analysis of these studies failed to show superior effects for CAT compared to IAT with regard to ADL ( $\mathrm{n}=193$; SMD, 0.17; 95\% CIs, -0.20 to $0.54, \mathrm{P}=0.37$; heterogeneity: $\chi^{2}=4.65, \mathrm{P}=0.20, \mathrm{I}^{2}=36 \%$ Figure $2 \mathrm{~B}$ ).

\section{Neurological Deficit Score (NDS)}

Two of the RCTs assessed the effects of EA-CAT compared to EA-IAT on NDS $[13,14]$. One trial showed the significant superior effects of EA-CAT [13], while the other one failed to do so[14]. A meta-analysis failed to 
Table 1 Key data from RCTs comparing contralateral acupuncture (CAT) to ipsilateral acupuncture (IAT) in post-stroke hemiplegic patients

\begin{tabular}{|c|c|c|c|c|c|c|c|c|}
\hline $\begin{array}{l}\text { First } \\
\text { author } \\
\text { (Year) }\end{array}$ & $\begin{array}{l}\text { Sample } \\
\text { size/ } \\
\text { Severity/ } \\
\text { Diagnosis }\end{array}$ & Groups & $\begin{array}{l}\text { Main } \\
\text { outcomes }\end{array}$ & $\begin{array}{l}\text { Intergroup } \\
\text { difference }\end{array}$ & CAT group & IAT group & $\begin{array}{l}\text { Co-interventions for } \\
\text { both groups }\end{array}$ & $\begin{array}{l}\text { Risk } \\
\text { of } \\
\text { bias* }\end{array}$ \\
\hline \multicolumn{9}{|c|}{ Infarction } \\
\hline $\begin{array}{l}\text { Pan } \\
\text { (2009) } \\
{[13]}\end{array}$ & $\begin{array}{l}53 \\
\text { n.r. } \\
\text { CT scan } \\
\text { or MRI }\end{array}$ & $\begin{array}{l}\text { (A) CAT } \\
(n=28) \\
(B) \text { IAT } \\
(n=25)\end{array}$ & $\begin{array}{l}\text { 1) } \\
\text { Response } \\
\text { rate } \\
\text { 2) NDS }\end{array}$ & $\begin{array}{l}\text { 1) } \mathrm{RR}, 1.16 \\
{[0.93,1.45] \text {, }} \\
\mathrm{NS} \\
\text { 2) } \mathrm{MD}, 3.69 \\
{[1.43,5.95]} \\
\mathrm{P}=0.001\end{array}$ & $\begin{array}{l}\text { PC6, LI4, ST36, LR3 } \\
\text { de-qi elicited, } \\
\text { manipulation at every } 5 \\
\text { min, } 20 \text { min per session, } \\
\text { once daily for } 10 \text { days, } \\
\text { interval of } 5 \text { days after } \\
\text { one course, three courses } \\
\text { in total }\end{array}$ & $\begin{array}{l}\text { Identical points and } \\
\text { procedures as CAT group }\end{array}$ & None & $\begin{array}{l}\text { U-U- } \\
\text { U-U- } \\
\text { Y-Y-Y }\end{array}$ \\
\hline $\begin{array}{l}\text { Chen } \\
(2007) \\
{[14]}\end{array}$ & $\begin{array}{l}68 \\
\text { Mild to } \\
\text { severe } \\
\text { CT scan } \\
\text { or MRI }\end{array}$ & $\begin{array}{l}\text { (A) EA CAT } \\
(n=34) \\
(B) \text { EA IAT } \\
(n=34))\end{array}$ & $\begin{array}{l}\text { 1) } \\
\text { Response } \\
\text { rate } \\
\text { 2) NDS }\end{array}$ & $\begin{array}{l}\text { 1) } \mathrm{RR}, 1.07 \\
{[0.88,1.31] \text {, }} \\
\mathrm{NS} \\
\text { 2) } \mathrm{MD}, 0.01 \\
{[-4.00,4.02] \text {, }} \\
\text { NS }\end{array}$ & $\begin{array}{l}\text { Points: LI15, LI11, LI10, TE5, } \\
\text { LI4, ST31, GB31, GB34, } \\
\text { ST36, ST41, GB36 } \\
\text { 1.7 Hz, for } 30 \text { min, once } \\
\text { daily }\end{array}$ & $\begin{array}{l}\text { Identical points and } \\
\text { procedures as CAT group }\end{array}$ & $\begin{array}{l}\text { Scalp acupuncture with } \\
\text { manual twirling at 180- } \\
200 \mathrm{~Hz} \text {, manipulation } \\
\text { at every } 10 \text { min for } 3 \\
\text { times, once daily for } 30 \\
\text { days }\end{array}$ & $\begin{array}{l}\text { U-U- } \\
U-U- \\
U-Y- \\
Y\end{array}$ \\
\hline $\begin{array}{l}\text { Liu } \\
(2005) \\
{[15]}\end{array}$ & $\begin{array}{l}60 \\
\text { Mild } \\
\text { (shoulder } \\
\text { pain) } \\
\text { CT scan } \\
\text { or MRI }\end{array}$ & $\begin{array}{l}\text { (A) Big } \\
\text { size } \\
\text { needle } \\
\text { CAT } \\
(n=30) \\
(B) \text { IAT } \\
(n=30)\end{array}$ & $\begin{array}{l}\text { Response } \\
\text { rate }\end{array}$ & $\begin{array}{l}\text { RR, } 1.17 \\
{[0.95,1.43]} \\
\text { NS }\end{array}$ & $\begin{array}{l}\text { Points: GB34 through } \\
\text { GB39 } \\
0.4-1.6 \mathrm{~mm} \times 150-500 \\
\text { mm, de-qi elicited, } \\
\text { manipulation at every } 10 \\
\text { min, for } 30 \mathrm{~min} \text {, once } \\
\text { daily, days n.r. }\end{array}$ & $\begin{array}{l}\text { Points: LI15, SI9, LI14, LI11, } \\
\text { TE5 } \\
0.30 \mathrm{~mm} \times 40 \mathrm{~mm} \text {, de-qi } \\
\text { elicited, manipulation at } \\
\text { every } 10 \mathrm{~min} \text {, for } 30 \mathrm{~min} \text {, } \\
\text { once daily, days n.r. }\end{array}$ & $\begin{array}{l}\text { Active and passive } \\
\text { exercises }\end{array}$ & $\begin{array}{l}\text { U-U- } \\
\text { N-N- } \\
\text { Y-Y- } \\
\text { U }\end{array}$ \\
\hline $\begin{array}{l}\text { Sun } \\
(2000) \\
{[16]}\end{array}$ & $\begin{array}{l}80 \\
\text { n.r. } \\
\text { CT scan } \\
\text { or MRI }\end{array}$ & $\begin{array}{l}\text { (A) CAT } \\
(n=40) \\
(B) \text { IAT } \\
(n=40)\end{array}$ & $\begin{array}{l}\text { Response } \\
\text { rate }\end{array}$ & $\begin{array}{l}\mathrm{RR}, 1.19 \\
{[1.00,1.41]} \\
P=0.05\end{array}$ & $\begin{array}{l}\text { Basic points: GV20, GB20, } \\
\text { LI15, LI11, TE5, LI4, GB34, } \\
\text { ST36, GB39, ST41 } \\
\text { Additional points: GV26, } \\
\text { PC8, LR3, BL18, BL23, BL17, } \\
\text { SP10, ST40, SP6 } \\
\text { De-qi elicited } \\
\text { One course: } 30 \text { min, once } \\
\text { daily for } 10 \text { sessions, } \\
\text { interval of } 2 \text { days after } \\
\text { one course } \\
\text { Three courses in total }\end{array}$ & $\begin{array}{l}\text { Identical points and } \\
\text { procedures as CAT group }\end{array}$ & None & $\begin{array}{l}\text { U-U- } \\
N-N- \\
Y-Y-Y\end{array}$ \\
\hline
\end{tabular}

Infarction and hemorrhage

\section{mixed}

\begin{tabular}{llll}
\hline Hong & 60 & (A) CAT & 1) \\
(2009) & Severe & $(\mathrm{n}=30)$ & Response \\
{$[17]$} & (post- & (B) IAT & rate \\
& stroke & $(\mathrm{n}=30)$ & 2) FMA \\
& shoulder- & & 3) ADL \\
& hand & & 4) Pain \\
& syndrome) & & (VAS) \\
& CT scan & & \\
& or MRI & &
\end{tabular}

$\begin{array}{llll}\mathrm{Ni} & 80 & \text { (A) CAT } & 1) \\ (2009) & \text { n.r. } & (\mathrm{n}=20) & \text { Response } \\ {[18]} & \text { CT scan } & \text { (B) IAT } & \text { rate } \\ & \text { or MRI } & (n=20) & \text { 2) FMA } \\ & & \text { (C) CAT } \\ & & + \text { CSS } \\ & (n=20)^{\dagger} \\ & & (D) \text { CSS } \\ & & (n=20)^{+}\end{array}$

1) $R R, 1.04 \quad 1^{\text {st }}$ set of points: LI15, SI9, $[0.89,1.21], \quad L I 10, T E 6, S I 3, G B 34$, and NS

2) $M D, 1.10 \quad 2^{\text {nd }}$ set of points: TE14,

$[0.06,2.14], \quad$ LI14, LI11, TE5, LI4, ST38,

$\mathrm{P}=0.04 \quad$ and most painful points

3) $M D, \quad \quad^{\text {st }}$ and $2^{\text {nd }}$ sets in turn,

$10.67[2.44, \quad 0.38 \mathrm{~mm} \times 40-65 \mathrm{~mm}$, de-

18.90], $P=$ qi elicited, $2 \mathrm{~Hz}$

0.01

4) $\mathrm{MD}$,

$-10.60$

$[-16.83$,

$-4.37], P=$

0.0009

1) $\mathrm{RR}, 1.33$

$[0.88,2.03]$

NS

2) $\mathrm{MD}$,

$14.54[9.42$,

19.66], $P<$

0.0001 manipulation at every 10

min,

One course: for $30 \mathrm{~min}$

once daily for 10 sessions,

interval of 2-3 days after

one course

Two courses in total

Points: PC6, LU5, LU4 for arms; ST36, GB34, LR3,

GB40, GB31 for legs,

taking turns every other

day

20 min, once daily for 6

days, 4 weeks in total
Identical points and None

procedures as CAT group

Y-U-

$\mathrm{N}-\mathrm{N}-$ Y-Y-Y
Points: L115, LI11, LI10, TE5, None Y-ULI4 for difficult extension; N-YLU5, PC3, PC6 for difficult flexion Identical procedures as CAT 
Table 1: Key data from RCTs comparing contralateral acupuncture (CAT) to ipsilateral acupuncture (IAT) in post-stroke hemiplegic patients (Continued)

\begin{tabular}{|c|c|c|c|c|c|c|c|c|}
\hline $\begin{array}{l}\text { Huang } \\
(2008) \\
{[19]}\end{array}$ & $\begin{array}{l}120 \\
\text { Mild to } \\
\text { severe } \\
\text { CT scan } \\
\text { or MRI }\end{array}$ & $\begin{array}{l}\text { (A) CAT } \\
(\mathrm{n}=30) \\
\text { strong } \\
\text { stimulation } \\
\text { (B) CAT } \\
(\mathrm{n}=30) \\
\text { weak } \\
\text { stimulation } \\
\text { (C) IAT } \\
(\mathrm{n}=30) \\
\text { strong } \\
\text { stimulation } \\
\text { (D) IAT } \\
\text { ( } \mathrm{n}=30 \text { ) } \\
\text { weak } \\
\text { stimulation }\end{array}$ & $\begin{array}{l}\text { 1) } \mathrm{FMA} \\
\text { 2) } \mathrm{ADL}\end{array}$ & $\begin{array}{l}\text { 1) A vs. C, } \\
\text { MD, } 2.50 \\
{[-2.43,7.43]} \\
\text { NS } \\
\text { B vs. D, } \\
\text { MD, }-0.60 \\
{[-5.78,4.58]} \\
\text { NS } \\
\text { 2) A vs. C, } \\
\text { MD, } 0.66 \\
{[-7.14,8.46]} \\
\text { NS } \\
\text { B vs. D, } \\
\text { MD, }-0.67 \\
{[-5.46,4.12]} \\
\text { NS }\end{array}$ & $\begin{array}{l}\text { LI15, LI14, TE10, TE9, TE5, } \\
\text { LI5, LI6, TE3 for arms; BL37, } \\
\text { LR9, ST36, GB39, BL62, } \\
\text { GB40, GB41 for legs } \\
30 \text { min, once daily for } 6 \\
\text { days, one day rest, for } 4 \\
\text { weeks } \\
\text { Twisting and twirling to } \\
180 \text { and } 90 \text { degrees/lifting } \\
\text { and thrusting to } 5 \text { and } 3 \\
\text { mm for strong and weak } \\
\text { stimulation, respectively }\end{array}$ & $\begin{array}{l}\text { HT1, LU5, PC3, LI11, LI10, } \\
\text { PC6, PC7, PC8 for arms; } \\
\text { ST31, ST32, BL40, BL57, } \\
\text { SP6, KI3, KI6, KI1 for legs } \\
\text { Identical procedures as } \\
\text { CAT group }\end{array}$ & None & $\begin{array}{l}Y-U- \\
N-N- \\
Y-Y-Y\end{array}$ \\
\hline $\begin{array}{l}\text { Seo } \\
(2001) \\
{[10]}\end{array}$ & $\begin{array}{l}13 \\
\text { Moderate } \\
\text { CT scan } \\
\text { or MRI }\end{array}$ & $\begin{array}{l}(\mathrm{A}) \text { CAT } \\
(\mathrm{n}=7) \\
(\mathrm{B}) \mathrm{IAT} \\
(\mathrm{n}=6)\end{array}$ & $\mathrm{MBI}$ & $\begin{array}{l}M D,-3.60 \\
{[-29.96} \\
22.76], N S\end{array}$ & $\begin{array}{l}\text { Points: GV20, CV24, GB20, } \\
\text { LI11, LI4, TE5, LI10, ST36, } \\
\text { GB34, GB31, GB39, LR3, } \\
\text { Bafeng, Baxie on } \\
\text { unaffected side; LI11, LI4, } \\
\text { ST36, LR3 on affected side } \\
20 \text { min, once daily for } 3 \\
\text { weeks }\end{array}$ & $\begin{array}{l}\text { Points: GV20, CV24, GB20, } \\
\text { LI11, LI4, TE5, LI10, ST36, } \\
\text { GB34, GB31, GB39, LR3, } \\
\text { Bafeng, Baxie on affected } \\
\text { side; LI11, LI4, ST36, LR3 } \\
\text { on unaffected side } \\
\text { Rest of procedures } \\
\text { identical as CAT group }\end{array}$ & None & $\begin{array}{l}U-U- \\
N-Y- \\
Y-Y-Y\end{array}$ \\
\hline
\end{tabular}

ADL: Activity of Daily Living Scale; AT: Acupuncture therapy; CAT: Contralateral acupuncture (needling the unaffected side); CSS: continual static stretch; CT: Computed tomography; FMA: Fugl-Meyer Assessment; IAT: Ipsilateral acupuncture (needling the affected side); min: minute; MBI: modified Barthel Index; MD: mean difference; n.a.: not applicable; MRI: Magnetic resonance imaging; NDS: Neurological Deficit Score; n.r.: not reported; NS: not significant; RR: response rate; VAS: visual analogue scale. ${ }^{+}$We excluded this group because it was not comparable to the other groups.

*(1) Was the allocation sequence adequately generated? (2) Was allocation adequately concealed? (3) Was knowledge of the allocated interventions adequately prevented during the study (both to patient and outcome assessor)? (4) Were incomplete outcome data adequately addressed? (5) Are reports of the study free of suggestion of selective outcome reporting? (6) Was the study apparently free of other problems that could put it at a risk of bias?; ( $\mathrm{Y}$ ) indicates "Yes (low risk of bias)"; (U), "Unclear"; (N), "No (high risk of bias)". ${ }^{12}$

show significant difference between the two methods ( $\mathrm{n}=121$; WMD, 2.23; $95 \% \mathrm{CIs},-1.35$ to $5.77, \mathrm{P}=0.22$; heterogeneity: $\chi^{2}=2.48, \mathrm{P}=0.12, \mathrm{I}^{2}=60 \%$ Figure $2 \mathrm{C}$ ).

\section{Motor function}

Three RCTs tested the effects of CAT for motor function on Fugl-Meyer Assessment (FMA) [17-19]. Two trials showed favorable effects of CAT $[17,18]$, while the other did not [19]. A meta-analysis of these studies failed to show superior effects for CAT compared to IAT ( $\mathrm{n}=220$; WMD, 6.70; 95\% CIs, -0.34 to $13.73, \mathrm{P}=$ $0.06)$ with heterogeneity $\left(\chi^{2}=20.88, \mathrm{P}=0.0 .001, \mathrm{I}^{2}=\right.$ $86 \%$ Figure 2D).

\section{Discussion}

Few rigorous RCTs testing the effects of CAT for poststroke rehabilitation are currently available, and the existing studies do not provide much information regarding the superiority of CAT over IAT for patients with post-stroke hemiplegia. Our meta-analysis of 6 trials demonstrated that CAT may be superior to IAT in post-stroke rehabilitation [13-18]. The total number of RCTs and total sample size included in our analysis, however, were too small to draw firm conclusions about the superiority of CAT.

Only four [13,16-18] of the 8 RCTs evaluated here reported favorable effects of CAT compared to IAT for at least one outcome measure. A meta-analysis showed that CAT had superior effects compared to IAT on response rate in patients with cerebral infarction [13-16] but failed to do so in a group of mixed patients with cerebral infarction or cerebral infarction $[17,18]$. Moreover, clinical trials should follow the CONSORT guidelines.

There are many prognostic factors for post-stroke rehabilitation, including type of stroke, size of the brain lesion, interval to onset, severity of symptoms, age, gender, and past history. Few of the trials included here, however, described these factors, and none of them conducted subgroup analysis for each prognostic factor. The stroke stage (acute or chronic) was also not reported. Outcomes were measured using ambiguous scales for many of the trials, despite the fact that it is important to select published or validated measurement scales. None of the trials conducted a follow-up assessment after treatment or reported adverse events or patient acceptance of acupuncture.

In the view of East-Asian Medicine, the Yellow Emperor Neijing states that, "if someone has disease related with the left side, the treatment point is the right side, and vice versa," emphasizing the importance of treatment side $[5,20]$. The clinical implications of this study may involve the selection of acupuncture points for treating stroke patients, which is thought to be one 


\section{(A) Response rate}

CAT IAT

Risk Ratio

Risk Ratio

\begin{tabular}{lrrrrrr} 
Study or Subgroup & Events & Total & Events & Total & Weight & M-H, Random, 95\% C \\
\hline $\mathbf{1 . 1 . 1}$ Cl & & & & & & \\
Chen 2007 & 30 & 34 & 28 & 34 & $16.6 \%$ & $1.07[0.88,1.31]$ \\
Liu 2005 & 28 & 30 & 24 & 30 & $15.9 \%$ & $1.17[0.95,1.43]$ \\
Pan 2009 & 26 & 28 & 20 & 25 & $13.3 \%$ & $1.16[0.93,1.45]$ \\
Sun 2000 & 38 & 40 & 32 & 40 & $22.5 \%$ & $1.19[1.00,1.41]$ \\
Subtotal (95\% Cl) & & $\mathbf{1 3 2}$ & & $\mathbf{1 2 9}$ & $\mathbf{6 8 . 3} \%$ & $\mathbf{1 . 1 5}[\mathbf{1 . 0 4}, \mathbf{1 . 2 7}]$
\end{tabular}

$122 \quad 104$

$1.15[1.04$

Heterogeneity: $\mathrm{Tau}^{2}=0.00 ; \mathrm{Chi}^{2}=0.65, \mathrm{df}=3(\mathrm{P}=0.88) ; \mathrm{I}^{2}=0 \%$

Test for overall effect: $Z=2.77(P=0.006)$

1.1.2 $\mathrm{Cl}$ and $\mathrm{ICH}$

Hong 2009

Ni 2009

Subtotal $(95 \% \mathrm{Cl})$

$28 \quad 30$

$16 \quad 20$

27

$30 \quad 28.0 \%$

Total events

44

50

$12 \quad 20 \quad 3.7 \%$

$50 \quad 31.7 \%$

$1.04[0.89,1.21]$

$1.33[0.88,2.03]$

$1.11[0.85,1.46]$

Heterogeneity: $\mathrm{Tau}^{2}=0.02 ; \mathrm{Chi}^{2}=1.78, \mathrm{df}=1(\mathrm{P}=0.18) ; \mathrm{I}^{2}=44 \%$

Test for overall effect: $Z=0.79(P=0.43)$

\begin{tabular}{lcccc} 
Total $(95 \% \mathrm{Cl})$ & \multicolumn{1}{c}{182} & 179 & $100.0 \%$ \\
Total events & 166 & 143 & \\
Heterogeneity: Tau $^{2}=0.00 ; \mathrm{Chi}^{2}=$ & $2.71, \mathrm{df}=5(\mathrm{P}=0.75) ; \mathrm{l}^{2}=0 \%$
\end{tabular}

Test for overall effect: $Z=2.80(P=0.005)$

\section{(B) Activities of Daily Living}

\begin{tabular}{|c|c|c|c|c|c|c|c|c|c|}
\hline \multirow[b]{2}{*}{ Study or Subgroup } & \multicolumn{3}{|c|}{ САТ } & \multicolumn{2}{|r|}{ IAT } & \multicolumn{3}{|r|}{ Std. Mean Difference } & \multirow{2}{*}{$\begin{array}{l}\text { Std. Mean Difference } \\
\text { IV. Random. } 95 \% \mathrm{Cl}\end{array}$} \\
\hline & Mean & SD & Total & Mean & SD & Total & Weight & IV. Random, $95 \% \mathrm{Cl}$ & \\
\hline Seo 2001 & 22.7 & 25.16 & 7 & 26.3 & 23.29 & 6 & $9.8 \%$ & $-0.14[-1.23,0.95]$ & \\
\hline Huang 2008b & 16 & 5.63 & 30 & 16.67 & 12.13 & 30 & $30.4 \%$ & $-0.07[-0.58,0.44]$ & \\
\hline Huang 2008a & 15.83 & 13.13 & 30 & 15.17 & 17.24 & 30 & $30.4 \%$ & $0.04[-0.46,0.55]$ & \\
\hline Hong 2009 & 43.67 & 16.13 & 30 & 33 & 16.38 & 30 & $29.4 \%$ & $0.65[0.13,1.17]$ & \\
\hline Total $(95 \% \mathrm{Cl})$ & & & 97 & & & 96 & $100.0 \%$ & $0.17[-0.20,0.54]$ & \\
\hline \multicolumn{9}{|c|}{$\begin{array}{l}\text { Heterogeneity: } \text { Tau }^{2}=0.05 ; \mathrm{Chi}^{2}=4.65, \mathrm{df}=3(P=0.20) ; \mathrm{I}^{2}=36 \% \\
\text { Test for overall effect: } Z=0.90(P=0.37)\end{array}$} & $\begin{array}{ccccc}-2 & -1 & 0 & 1 & 2 \\
\text { Favours } & \text { IAT } & \text { Favours } & \mathrm{C} A\end{array}$ \\
\hline
\end{tabular}

(C) Neurological Deficit Score

\begin{tabular}{|c|c|c|c|c|c|c|c|c|c|}
\hline \multirow[b]{2}{*}{ Study or Subgroup } & \multicolumn{2}{|r|}{ CAT } & \multicolumn{3}{|r|}{ IAT } & \multirow[b]{2}{*}{ Total } & \multirow[b]{2}{*}{ Weight } & \multirow{2}{*}{$\begin{array}{l}\text { Mean Difference } \\
\text { IV. Random, } 95 \% \mathrm{Cl}\end{array}$} & \multirow{2}{*}{$\begin{array}{c}\text { Mean Difference } \\
\text { IV, Random, } 95 \% \mathrm{Cl}\end{array}$} \\
\hline & Mean & SD & Total & Mean & SD & & & & \\
\hline Chen 2007 & 7.96 & 8.29 & 34 & 7.97 & 8.59 & 34 & $39.6 \%$ & $-0.01[-4.02,4.00]$ & \\
\hline Pan 2009 & 12.57 & 5.02 & 28 & 8.88 & 3.28 & 25 & $60.4 \%$ & $3.69[1.43,5.95]$ & \\
\hline Total $(95 \% \mathrm{Cl})$ & & & 62 & & & 59 & $100.0 \%$ & $2.23[-1.32,5.77]$ & \\
\hline \multicolumn{9}{|c|}{ Heterogeneity: $\mathrm{Tau}^{2}=4.08 ; \mathrm{Chi}^{2}=2.48, \mathrm{df}=1(\mathrm{P}=0.12) ; \mathrm{I}^{2}=60 \%$} & $\begin{array}{cccc}-10 & -5 & 0 & 5\end{array}$ \\
\hline
\end{tabular}

\section{(D) Motor function}

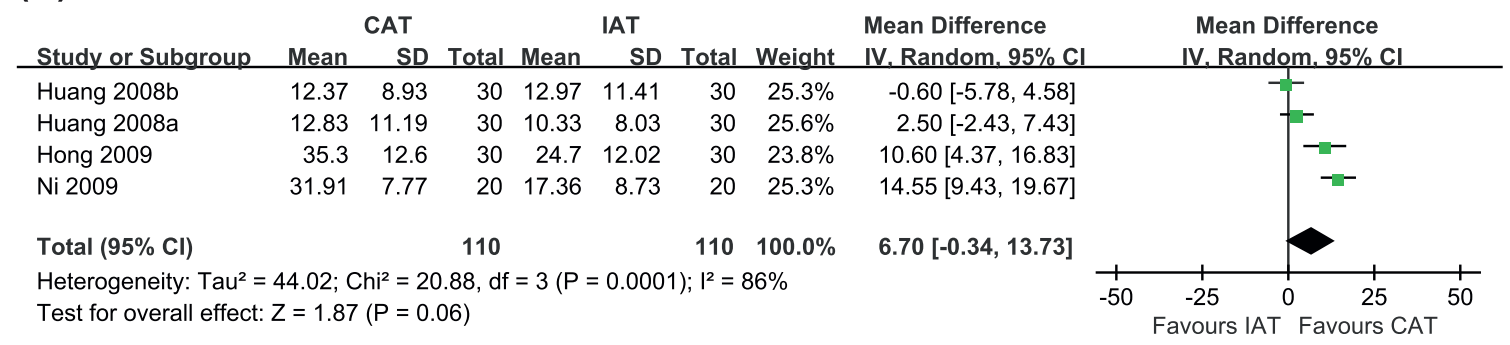

Figure 2 Forest plot comparing contralateral acupuncture (CAT) to ipsilateral acupuncture (IAT) in terms of (A) response rate, (B) Activities of Daily Living, (C) Neurological Deficit Score, and (D) motor function in post-stroke hemiplegic patients. Cl: cerebral infaction; $\mathrm{ICH}$ : intracranial hemorrhage. 
of the key issues in therapeutic effectiveness of acupuncture. In this review, there was a significant difference between CAT and IAT in terms of response rate; however, high risk of bias in the included trials prevents us from making firm conclusions. Not a single trial in our review adequately concealed group allocation and 5 out of 8 trials did not clearly reported how randomization was conducted. This raises concerns about validity of the results from these trials hence limits their applicability. In comparison of CAT with IAT in post-stroke hemiplegia, it is almost impossible to blind patients as demonstrated in our risk of bias assessment. Future trials then should pay more attention to adopting blinded outcome evaluation using validated scales rather than arbitrarily categorized response rate. One interesting finding of our review is that there was no between group difference in ADL or motor function on FMA while response rate in CAT was significantly better than IAT. It is not difficult to attribute this finding to failed blinding of included trials.

Assuming that CAT is beneficial for post-stroke rehabilitation, its mechanism of action may be of interest. The postulated modes of action involve changes in brain activity according to treatment side [20] and direct effects on target organs and the central nervous system [21-23]. None of these theories, however, has been established in the literature.

This systematic review has several limitations. First, relevant RCTs may have been overlooked despite our best efforts to conduct sensitive literature searches without language restrictions. Second, all included RCTs were conducted in China and Korea, and studies from these countries are known to exhibit a very low rate of negative results [24]. In fact, the distorting effects of publication bias and location bias have been well-documented $[25,26]$. Finally, the reviewed studies had a high risk of bias; therefore, the results of this review might be exaggerated. Methodological shortcomings such as inadequate allocation concealment, small sample size and inadequate blinding require further large studies that incorporate methodological rigor.

\section{Conclusion}

The results of our systematic review and meta-analysis suggest that there is limited evidence of CAT being superior to IAT in post-stroke rehabilitation. The paucity of RCTs included in our analysis, coupled with small sample size and high risk of bias, prevents us from drawing firm conclusions about the effectiveness of CAT. Future rigorous studies are required to confirm our limited findings.

\section{Conflicts of interests}

The authors declare that they have no competing interests.

\section{Acknowledgements}

This study was supported by a grant of the Oriental Medicine R\&D Project, the Ministry of Health and Welfare (MOHW), Republic of Korea [No. B080053].

\section{Author details}

${ }^{1}$ Department of Korean Internal Medicine, Dongguk University Ilsan Hospital, Goyang, South Korea. ${ }^{2}$ Department of Oriental Medicine, Dongguk University Graduate School, Seoul, South Korea. ${ }^{3}$ Division of Standard Research, Korea Institute of Oriental Medicine, Daejeon, South Korea. ${ }^{4}$ Acupuncture and Meridian Science Research Center, College of Oriental Medicine, Kyung Hee University, Seoul, South Korea.

\section{Authors' contributions}

MKK and $\mathrm{CHH}$ conceived the study design. MKK, TYC and MSL searched and selected the trials and extracted, analyzed and interpreted the data. MKK and MSL drafted the manuscript. TYC and HSL updated the search and the content of the review. MSL, HSL and CHH helped with the study design and critically reviewed the manuscript. All authors read and approved the final version of the manuscript.

Received: 28 March 2010 Accepted: 30 July 2010

Published: 30 July 2010

\section{References}

1. Andrews AW, Bohannon RW: Distribution of muscle strength impairments following stroke. Clin Rehabil 2000, 14(1):79-87.

2. Brown RD Jr, Ransom J, Hass S, Petty GW, O'Fallon WM, Whisnant JP, Leibson CL: Use of nursing home after stroke and dependence on stroke severity: a population-based analysis. Stroke 1999, 30(5):924-929.

3. Ernst $\mathrm{E}$, Pittler MH, Wider B, Boddy $\mathrm{K}$ : The desktop guide to complementary and alternative medicine Philadelphia, PA: Mosby Elservier 2006.

4. Shah SH, Engelhardt R, Ovbiagele B: Patterns of complementary and alternative medicine use among United States stroke survivors. J Neurol Sci 2008, 271(1-2):180-185.

5. Yellow Emperor: Yellow Emperor's Inner Classic of Internal Medicine, Spiritual Pivot (Ling Shu). Seoul: Euisungdang publishing 2002, 214.

6. Kim KS, Kim MS, Park BR, Park SK, Sohn IC, Kim JH: Effects of hetero segmental electro - acupuncture on formalin induced pain in the rat. $J$ Meridian Acupoint 2000, 17(2):231-246.

7. Kim YS, Moon SK, Park SU, Han C: Integrated clinical approach to stroke. Seoul: Jung Dam Pub 2007.

8. Cheng B: 206 cases of spinogenic dizziness treated by contralateral acupuncture. J Tradit Chin Med 1996, 16(1):35-36.

9. Lu F: Clinical application of contralateral acupuncture technique. J Tradit Chin Med 1997, 17(2):124-126.

10. Seo JC, Cheong BS, Yun HS, Cho SG, Kim YM, Kim Jl, Lee YH: Effects of contralateral acupuncture on recovery of motor disorders in stroke patients. J Meridian Acupoint 2001, 18(3):1-9.

11. Wu P, Mills E, Moher D, Seely D: Acupuncture in poststroke rehabilitation: a systematic review and meta-analysis of randomized trials. Stroke 2010, 41(4):e171-179.

12. Higgins JPT, Douglas GA: Assessing Risk of Bias in Included Studies. Cochrane Handbook for Systematic Reviews of Interventions West Sussex, England: Wiley-BlackwellJulian PTH, Green S 2008, 187-241.

13. Pan Z: Clinical efficacy of giant acupuncture treatment of ischemic stroke. Doctorial thesis, Guangzhou Univesity of Chinese Medicine 2009.

14. Chen $X$ : Scalp acupuncture plus contralateral acupuncture treatment for cerebral infarction with hemiplegic patients. Chin J Rehabil 2007, 22(2):120.

15. Liu G: Great needle of contralateral acupuncture treatment for poststroke shoulder pain and hemorheology. Chin J Integr Med Cardio-/ Cerebrovasc Dis 2005, 3(6):538-539.

16. Sun Y: Contralateral acupuncture treatment of 40 cases for cerebral infarction. Heilongjiang J Tradit Chin Med 2000, 1:57-58.

17. Hong J, Sheng $P$, Yuan $Y$, Y Y S: Observation on therapeutic effect of opposing needling for treatment of poststroke shoulder-hand syndrome. Chin Acupunct Moxibusion 2009, 29(3):205-208.

18. Ni K, Luo Y, Wang Y, Ge F, Chen SX, Chu JM, Shu GY: Clinical observation of recuperative effect of opposing needling accompanying continual 
static stretch in treating high muscular tension of apoplectic hemiplegia. J Zhejiang Univ Tradit Chin Med 2009, 33(4):576-577.

19. Huang Z: The clinical study of hemiplegia spasm after a stroke treated by acupunctureing the points in spasm side and non-spasm side. Doctorial thesis, Guangzhou Univesity of Chinese Medicine 2008.

20. Woo YM, Lee MS, Nam Y, Cho HJ, Shin BC: Effects of contralateral electroacupuncture on brain function: a double-blind, randomized, pilot clinical trial. J Altern Complement Med 2006, 12(8):813-815.

21. Ueda $Y$, Hayashi $K$, Kuriowa $K$ : The application of fMRI to basic experiments in acupuncture. The effects of stimulus points and content on cerebral activities and responses. IEEE Eng Med Biol Mag 2005, 24(2):47-51

22. Lo YL, Cui SL: Acupuncture and the modulation of cortical excitability. Neuroreport 2003, 14(9):1229-1231.

23. Hui KK, Liu J, Makris N, Gollub RL, Chen AJ, Moore Cl, Kennedy DN, Rosen BR, Kwong KK: Acupuncture modulates the limbic system and subcortical gray structures of the human brain: evidence from fMRI studies in normal subjects. Hum Brain Mapp 2000, 9(1):13-25.

24. Vickers A, Goyal N, Harland R, Rees R: Do certain countries produce only positive results? A systematic review of controlled trials. Control Clin Trials 1998, 19(2):159-166.

25. Ernst E, Pittler MH: Alternative therapy bias. Nature 1997, 385(6616):480.

26. Rothstein HR, Sutton AJ, Borenstein M: Publication bias in meta-analysis. Publication bias in meta-analysis Chichester, West Sussex: WileyRothstein HR, Sutton AJ, Borenstein M 2005.

Pre-publication history

The pre-publication history for this paper can be accessed here: http://www.biomedcentral.com/1472-6882/10/41/prepub

doi:10.1186/1472-6882-10-41

Cite this article as: Kim et al.: Contralateral acupuncture versus ipsilateral acupuncture in the rehabilitation of post-stroke hemiplegic patients: a systematic review. BMC Complementary and Alternative Medicine 2010 10:41.

\section{Submit your next manuscript to BioMed Central and take full advantage of:}

- Convenient online submission

- Thorough peer review

- No space constraints or color figure charges

- Immediate publication on acceptance

- Inclusion in PubMed, CAS, Scopus and Google Scholar

- Research which is freely available for redistribution 